\title{
Existence of finite rigidity layer at the base of the Earth's liquid outer core inferred from anomalous splitting of normal modes
}

\author{
Seiji Tsuboi ${ }^{1,2}$ and Masanori Saito ${ }^{1}$ \\ ${ }^{1}$ Graduate School of Integrated Science, Yokohama City University, Yokohama 236-0027, Japan \\ ${ }^{2}$ Institute for Frontier Research on Earth Evolution, Japan Marine Science Technology Center, Yokosuka 237-0061, Japan
}

(Received June 25, 2001; Revised January 4, 2002; Accepted January 4, 2002)

\begin{abstract}
We calculate normal modes for the Earth model, which has a slight rigidity layer at the base of the liquid outer core. We show that such a layer with thickness about $40 \mathrm{~km}$ and the shear wave velocity of $0.017 \mathrm{~km} / \mathrm{sec}$ can produce a normal mode, which has a close eigenfrequency to that of liquid core model, without affecting fundamental modes and most of the higher modes. Our results indicate that the thin finite rigidity layer at the base of the outer core might explain the anomalous splitting of the Earth's normal modes, which has not been fully explained by the anisotropy in the inner core.
\end{abstract}

\section{Introduction}

The nature of an anomalous splitting of the Earth's normal modes, which is characterized as a spectral splitting width of several normal modes is almost twice as large as that is predicted by the rotation and hydrostatic ellipticity of the Earth (Masters and Gilbert, 1981; Ritzwoller et al., 1986; Giardini et al., 1987), has been controversial for more than a decade (Dahlen and Tromp, 1998). It is now considered that the anomalous splitting can be explained by the inner-core anisotropy (Woodhouse et al., 1986; Tromp, 1993; Dahlen and Tromp, 1998), because the inner-core anisotropy obtained from normal modes seems to be consistent to those obtained from travel time observations (Poupinet et al., 1983; Creager, 1992). However, there still remain core sensitive modes which are not fully explained by the inner-core anisotropy, such as ${ }_{3} S_{2}$. The splitting width of ${ }_{3} S_{2}$ predicted by the inner-core anisotropy is much larger than that of the observation. Based on this observation, it is argued that there exists a heterogeneous structure in the Earth's liquid outer core (Widmer et al., 1992; Romanowicz and Breger, 1999; Romanowicz et al., 2000).

Although it is difficult to introduce heterogeneity of compressional wave velocity and density in the liquid outer core, finite rigidity in the outer core might explain anomalous splitting of such core sensitive modes. Sato (1964) has shown that if we allow slight rigidity in the liquid core, the modes which have nearly equal eigenfrequencies to that of a liquid core model would appear. Since this is similar to the spectrum splitting of normal modes, he called this as "soft core splitting." Here, we consider the Earth model which has an extremely slow shear velocity layer at the bottom of the liquid outer core. We show that such a layer with thickness about $40 \mathrm{~km}$ and the shear wave velocity of 0.017

Copy right (C) The Society of Geomagnetism and Earth, Planetary and Space Sciences (SGEPSS); The Seismological Society of Japan; The Volcanological Society of Japan; The Geodetic Society of Japan; The Japanese Society for Planetary Sciences. $\mathrm{km} / \mathrm{sec}$ can actually produce soft core splitting modes for core sensitive modes, such as ${ }_{3} S_{2}$, without affecting fundamental modes and most of the higher modes.

\section{Soft Core Splitting}

It is argued that there may be some heterogeneous structure of the compressional velocity or the density in the liquid outer core (Widmer et al., 1992; Romanowicz and Breger, 1999; Romanowicz et al., 2000), because those modes, which show anomalous splittings, are sensitive to the structure of the outer core. Heterogeneous structure in the outer core is required if we should explain the anomalous splitting by the splitting of isolated normal modes, although the general idea that the liquid outer core should be homogeneous has ruled out this possibility. However, there may be another possibility that spherically symmetric structure in the outer core may produce splitting-like behavior of normal modes. Sato (1964) has calculated torsional oscillation of an earth model consisting of a homogeneous mantle and a slightly rigid homogeneous core. He showed that if there is a finite rigidity in liquid core with several percent of the mantle, there appear eccentric modes which have nearly equal frequencies to those of a liquid core model. Since these modes appear in the spectrum with nearly equal amplitudes and frequencies, this may be considered as a kind of spectrum splitting. Sato has called these phenomena as "soft core splitting."

Finite rigidity with several percent of the mantle should be impossible to be introduced throughout in the Earth's liquid core, because the agreement of the observed eigenfrequencies of fundamental and higher modes with those predicted by the spherically symmetric Earth model with liquid outer core is excellent (Gilbert and Dziewonski, 1975; Dziewonski and Anderson, 1981). However, it may be possible to have a thin finite rigidity layer in the liquid outer core without affecting most of the normal modes except those sensitive to outer core structure. It is shown 
that the anomalous splitting is not observed for torsional modes (Tromp and Zanzerkia, 1995), which implies that finite rigidity layer should not exist below the core-mantle boundary. Here we calculate spheroidal modes for Earth model with slight rigidity in just above the inner core boundary and examine if there exists "soft core splitting."

We use isotropic PREM (Dziewonski and Anderson, 1981) as spherically symmetric Earth model and introduce slight shear wave velocity at the lowermost layer of the outer core. The system of ordinary differential equations governing the Earth's spheroidal oscillation is of sixth order in solid regions and of fourth order in fluid regions (Takeuchi and Saito, 1972). Traditionally, either Runge-Kutta-Gill method (Saito, 1988; Woodhouse, 1988) or Rayleigh-Ritz variational method (Wiggins, 1976; Buland, 1988) has been used to solve this system. We use program package DISPER80 (Saito, 1988), which uses Runge-Kutta-Gill method, to obtain eigenfrequency and eigenfunction of 'soft core' modes. The radial eigenfunction of these normal modes oscillate severely within the slow shear wave velocity layer in the outer core. Since DISPER80 uses fixed step Runge-KuttaGill method to integrate eigenfunction in the radial direction, we need to have as fine radial grid size of model parameter as is possible in this layer to keep good accuracy. We check a wavelength of the radial eigenfunction in this layer and try to keep a radial grid of the model parameter about one hundredth of the wavelength. DISPER80 uses two parameters to check accuracy of eigenfrequencies and eigenfunctions. One is the ordinally Rayleigh quotient and the other is a kinetic energy computed by different formula (Saito, 1988). We have checked these parameters to make sure that the results are in good accuracy. We believe that by doing this we can get good accuracy for both eigenfrequencies and eigenfunctions up to period about 300-400 second. Those modes with much shorter periods should be calculated by using much sophisticated scheme. In the present paper, we use two "soft core" Earth models: (1) layer thickness $126 \mathrm{~km}$ and constant shear wave velocity of $0.005 \mathrm{~km} / \mathrm{sec}$ in the bottom of the liquid outer core (model 1) and (2) layer thickness $40 \mathrm{~km}$ and constant shear wave velocity of 0.0171 $\mathrm{km} / \mathrm{sec}$ in the bottom of the liquid outer core (model 2).

\section{Normal Modes for Soft Core Earth Model}

We calculate the eigenfrequency and the eigenfunction of ${ }_{0} S_{2}$ for "soft core" Earth models to see if there exists "soft core" mode. The radial eigenfunctions of this ${ }_{0} S_{2}$ equivalent mode are shown in Fig. 1. The period of this mode is $3233.26 \mathrm{sec}$ for model 1 and $3231.63 \mathrm{sec}$ for model 2, while that predicted by isotropic PREM with a liquid core is $3233.45 \mathrm{sec}$ and the observation is $3233.25 \mathrm{sec}$. The radial eigenfunctions for both of the models oscillate severely within the finite rigidity layer. The wavelength of the radial eigenfunction within the finite rigidity layer is about $6 \mathrm{~km}$ for both of the models. However, the period of these modes do not differ significantly from both observation and that predicted by the liquid core model. There are no soft core splitting modes for ${ }_{0} S_{2}$. Thus, this order of finite rigidity in the liquid outer core does not affect ${ }_{0} S_{2}$. Fundamental spheroidal modes with the angular order greater than 8 (period $707 \mathrm{sec}$ ) do not have sensitivity to the inner core bound-
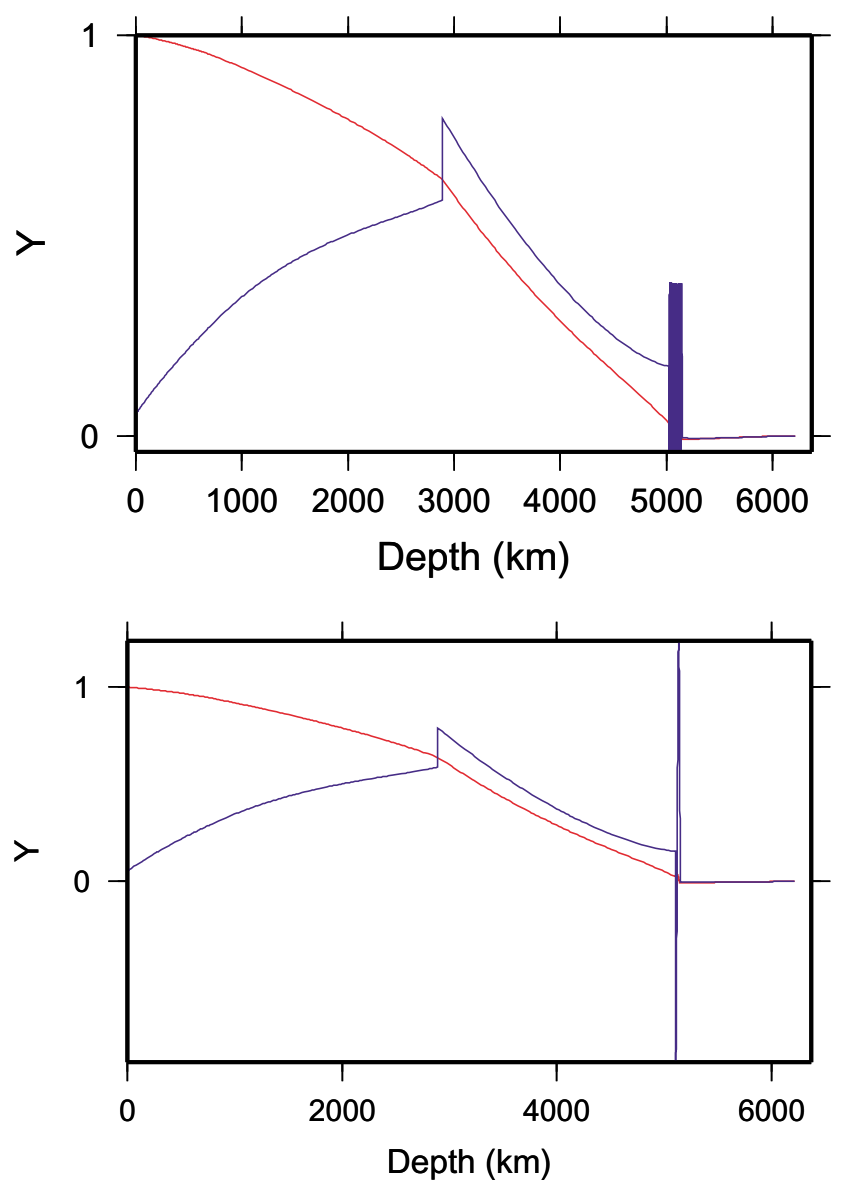

Fig. 1. Radial eigenfunctions of ${ }_{0} S_{2}$ for soft core Earth model. Red: vertical displacement $y_{1}(r)$ (Saito, 1988), Blue: horizontal displacement $y_{3}(r)$ (Saito, 1988). Discontinuites in the eigenfunctions indicate the core-mantle boundary (at depth $2891 \mathrm{~km}$ ) and the inner core-outer core boundary (at depth $5149.5 \mathrm{~km}$ ). Eigenfunctions are shown for model 1 (top) and model 2 (bottom). The period of the mode for model 1 is $3233.26 \mathrm{sec}$ and for model 2 is $3231.63 \mathrm{sec}$.

ary structure. We have calculated fundamental modes up to the angular order 8 and found that the eigenfrequencies are not affected and there are no soft core splitting modes. We also have calculated the first higher spheroidal modes up to the angular order 6 (period $657 \mathrm{sec}$ ) for the same soft core Earth models. There are no soft core splitting modes for these higher modes, which shows that these first higher modes are not affected by slight shear wave velocity layer at the bottom of the outer core. We may conclude that this order of finite rigidity may be introduced at the bottom of the liquid outer core without affecting most of the fundamental and higher modes.

\section{Results for Anomalously Splitted Modes}

We have calculated, then, the normal modes of anomalously splitted spheroidal mode, ${ }_{3} S_{2}$, which is not still fully explained by the inner core anisotropy. We have found that both of the models produce two soft core splitting modes for ${ }_{3} S_{2}$ as Sato predicted. The radial eigenfunctions of these modes are shown in Figs. 2 and 3. For model 1, one mode has a similar shape of eigenfunction to that of the liquid core model with the period of $903.87 \mathrm{sec}$. The other has basically the same eigenfunction but large amplitude within the finite 

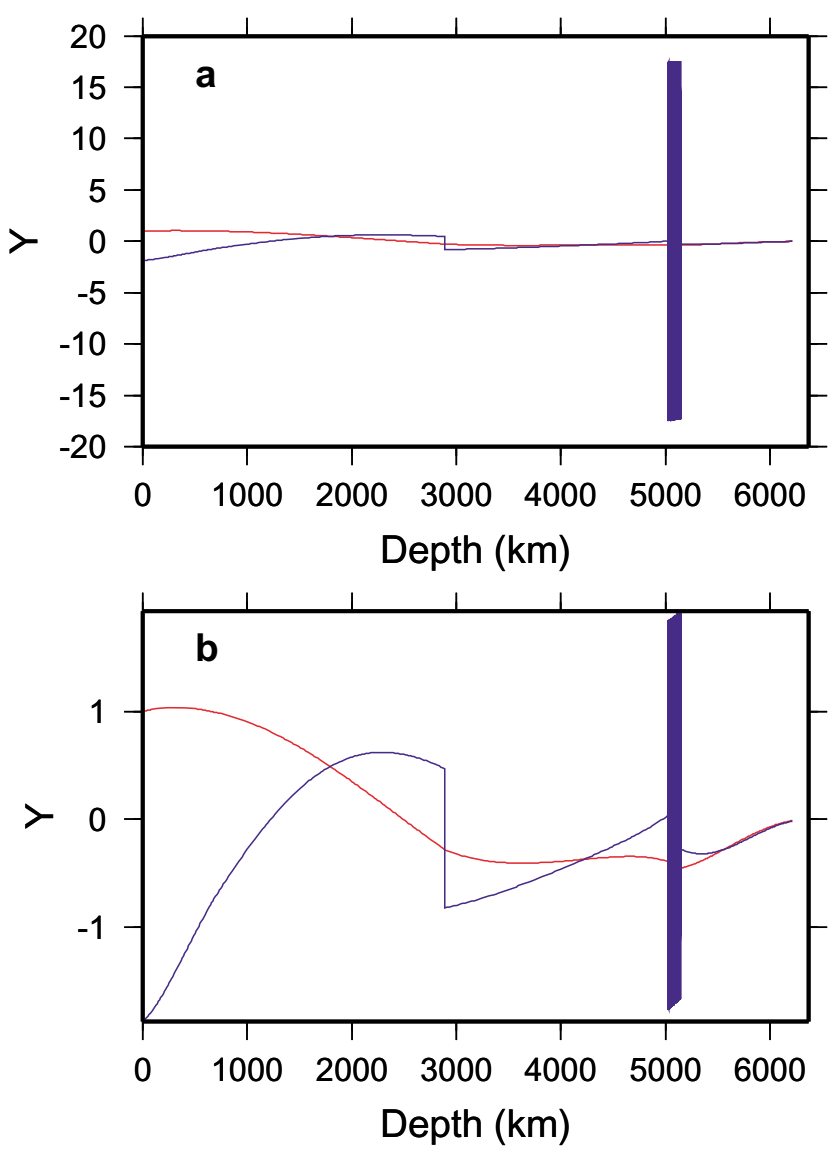

Fig. 2. The same as Fig. 1 but for ${ }_{3} S_{2}$ for soft core Earth model 1. The period of the mode is (a) $905.16 \mathrm{sec}$ and (b) $903.87 \mathrm{sec}$.

rigidity layer in the outer core with the period of $905.16 \mathrm{sec}$. There are only these two soft core splitting modes for ${ }_{3} S_{2}$ equivalent mode. For model 2, there are also two modes, of which period is $902.52 \mathrm{sec}$ and 907.33 . The anomalous splitting width of ${ }_{3} S_{2}$ is reported to be between about 904 sec and $912 \mathrm{sec}$. The liquid core model predicts the period should be $904 \mathrm{sec}$ and the splitting width due to the Earth's rotation and elliptical figure should be between about 902 and $907 \mathrm{sec}$. Since the eigenfunctions of soft core splitting modes have the same characteristics as those of the liquid core model, we may assume that they have the same rotational and elliptical splitting parameters. Then the total splitting width of these soft core splitting modes may be between 902 and $908 \mathrm{sec}$ for model 1 and 900 and 910 for model 2 . Thus it is possible that the "soft core splitting" may explain the observed splitting width of ${ }_{3} S_{2}$. The amplitude of eigenfunction within the finite rigidity layer of the mode with the period of $905.16 \mathrm{sec}$ for model 1 is about one twentieth to the amplitude near the Earth's surface. It is not necessarily large but is sufficient to be excited by the earthquakes that occurred near the Earth's surface. However, since the eigenfunction should be normalized by the kinetic energy integral, the size of the kinetic energy is a good measure of the amplitude of excited modes. While the kinetic energy of the mode with the period of $903.87 \mathrm{sec}$ is 2.613 , the kinetic energy of the mode with the period of $905.16 \mathrm{sec}$ is 25.096 for model 1 . Therefore, it is not likely that the mode with the
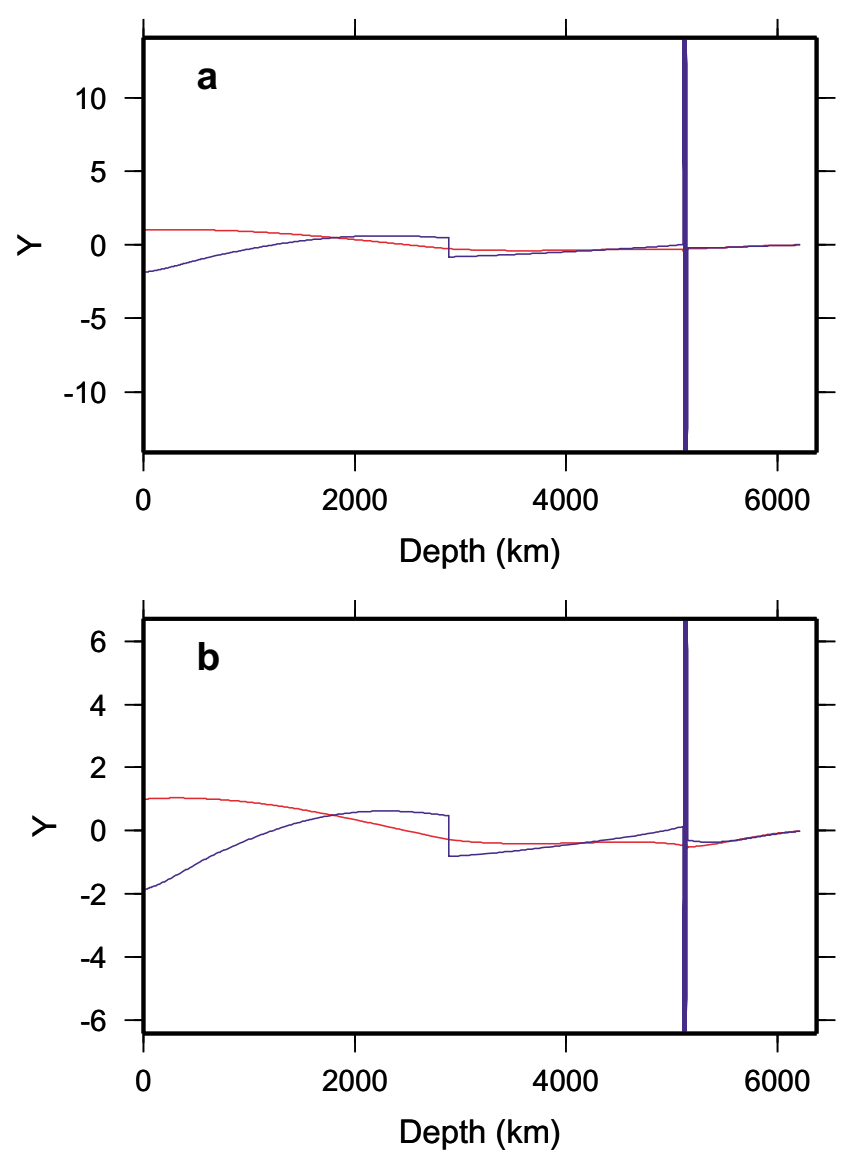

Fig. 3. The same as Fig. 1 but for ${ }_{3} S_{2}$ for soft core Earth model 2. The period of the mode is (a) $907.33 \mathrm{sec}$ and (b) $902.52 \mathrm{sec}$.

period $905.16 \mathrm{sec}$ for model 1 affects the spectrum of ${ }_{3} S_{2}$. On the other hand, for model 2, the kinetic energy of the mode with the period of $902.52 \mathrm{sec}$ is 3.528 and the mode with $907.33 \mathrm{sec}$ is 7.138 , which shows that these 2 modes can be identified in the spectrum.

Our results indicate that finite rigidity layer with thickness $40 \mathrm{~km}$ and constant shear wave velocity of $0.0171 \mathrm{~km} / \mathrm{sec}$ in the bottom of the liquid outer core (model 2) can be a possible candidate to explain anomalous splitting of ${ }_{3} S_{2}$. We also have calculated ${ }_{2} S_{3}$ equivalent modes for soft core earth models. It is already shown that the anomalous splitting of ${ }_{2} S_{3}$ can be explained by the inner core anisotropy. We found that two soft core splitting modes of ${ }_{2} S_{3}$, of which periods are $804.99 \mathrm{sec}$ and $805.50 \mathrm{sec}$, exist for model 1, but no soft core splitting modes exist for model 2 . Thus we may consider that this thin finite rigidity layer assumed in our model 2 affects only the modes which is really sensitive to the inner core boundary structure, such as ${ }_{3} S_{2}$. Previous studies have shown that the anomalous splitting is basically explained by the inner core anisotropy, except for some peculiar modes such as ${ }_{3} S_{2}$. Then, it is necessary to introduce other explanation for some modes, such as ${ }_{3} S_{2}$. Our results imply that the soft core splitting caused by thin finite rigidity layer assumed in our model 2 can be one candidate, since the soft core splitting exists for ${ }_{3} S_{2}$ but not for ${ }_{2} S_{3}$. Recent study by Dziewonski and Ishii (2001) has shown that both the body wave travel time anomaly and the normal modes can be ex- 

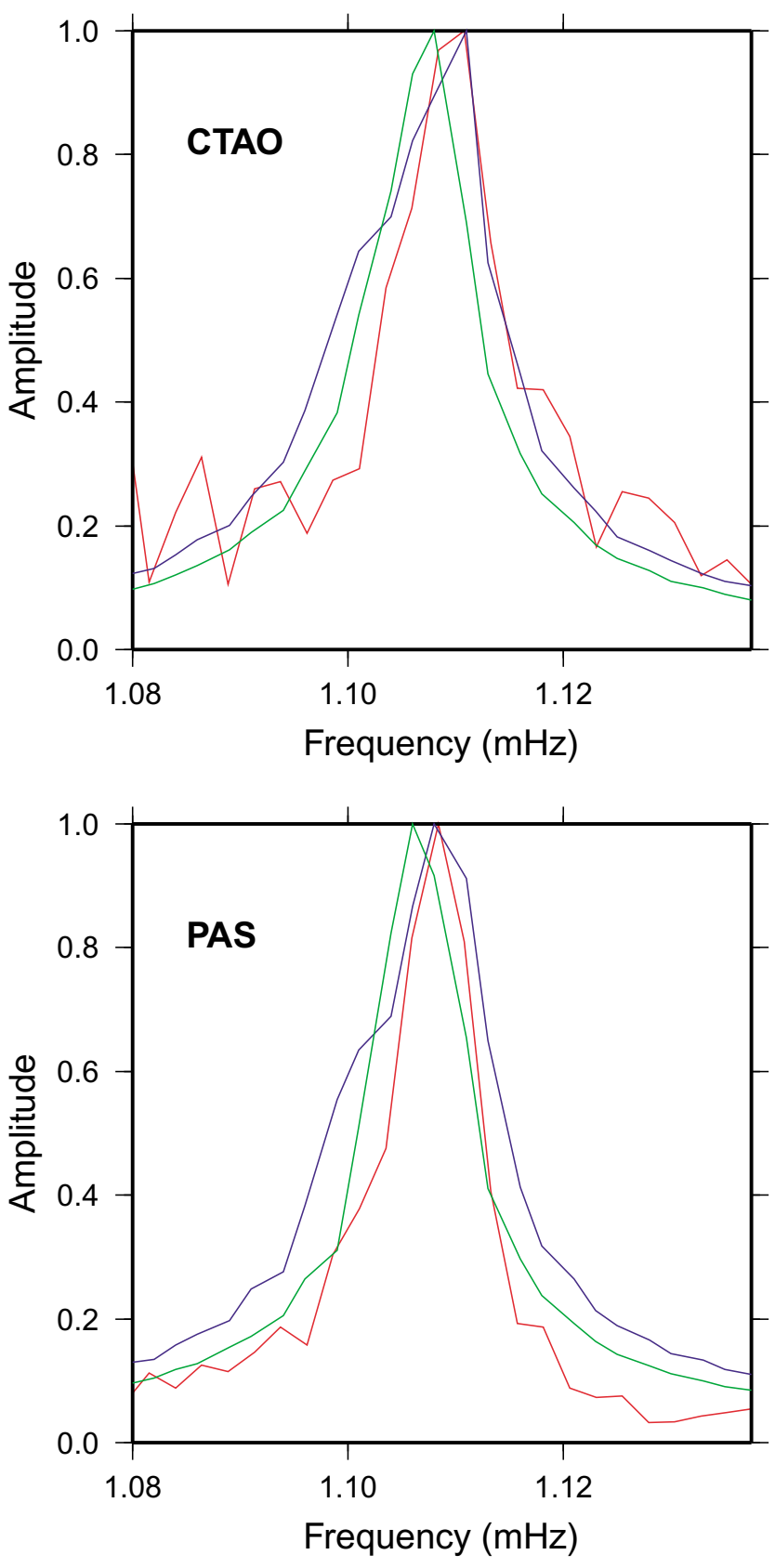

Fig. 4. The synthetic and observed spectrum of ${ }_{3} S_{2}$ for June 9, 1994 Bolivian earthquake. 80 hours of the vertical component velocity seismograms of UHZ channel are Hanning tapered before calculating the Fourier spectrum. Each spectrum is normalized by the peak amplitude. Red: observed spectrum, Green: synthetic spectrum for liquid core model with rotational and elliptical splitting included, Blue: synthetic spectrum for soft core model with rotational and elliptical splitting included. (top) station CTAO and (bottom) station PAS.

plained by the weak anisotropy in the inner core. They suggest that the travel time anomaly, which does not fit to their simple model, is caused by some regional structure in the core-mantle boundary region. We may consider that our results may be consistent to their suggestion, because our results imply that the anomalous splitting may be explained by the inner core anisotropy but ${ }_{3} S_{2}$ may be explained by soft core splitting due to thin finite rigidity layer at the bottom of the liquid outer core, which does not affect other modes such as ${ }_{2} S_{3}$.

\section{Synthetic Spectrum}

We, then, calculate synthetic spectrum of these ${ }_{3} S_{2}$ equivalent soft core splitting modes excited by June 9, 1994 Bolivian earthquake and compare with the observed spectrum. We arbitrarily select stations Charters Towers (CTAO) and Pasadena (PAS) from IRIS FARM volumes of this event. We use a moment tensor solution obtained from Harvard CMT catalog and calculate 80 hours synthetic vertical ground velocity records for these two stations. We calculate synthetics for both soft core Earth model and liquid core Earth model. In both cases, we assume the same rotational and elliptical splitting parameters to get eigenfrequencies of ten singlets for soft core case and 5 singlets for liquid core case. We also assume that the attenuation factor of each singlet is the same as that of the multiplet calculated for the liquid core model.

Results are shown in Fig. 4 with the observed spectrum. Since, there are a factor of 2-3 differences between the observed amplitude of spectral peak and the synthetics because of possible ambiguity in the moment tensor solution, we normalized the each spectrum with its peak amplitude and compare the overall spectral shape. Apparently, the spectral peak width predicted by the liquid core model, which includes only rotational and elliptical splitting, is smaller than the observation. Especially, the small bump appeared in the observation around $1.1 \mathrm{mHz}$ is not modeled. On the other hand, the synthetics computed for soft core model reproduce these characteristics well because of the soft core splitting mode shown in Fig. 3. Thus, Fig. 4 shows that the soft core splitting has a potential to explain anomalous splitting of ${ }_{3} S_{2}$.

\section{Conclusion}

We have calculated normal modes for the Earth model which has slight rigidity in the bottom of the liquid outer core. Our preliminary calculation suggests that the slight rigidity layer with the thickness $40 \mathrm{~km}$ and the shear wave velocity of $0.0171 \mathrm{~km} / \mathrm{sec}$ is compatible with the fundamental modes and most of the higher modes. We found that coresensitive mode, such as ${ }_{3} S_{2}$, has soft core splitting modes, which may explain anomalous splitting. This result indicates that the slight rigidity in the liquid outer core can be considered as a candidate to explain anomalous splitting of core sensitive modes.

Acknowledgments. We would like to express our gratitude to two anonymous reviewers for their constructive comments regarding this work.

\section{References}

Buland, R., Variational Methods, in Seismological Algorithms: Сотриtational Methods and Computer Programs, edited by D. J. Doornbos, pp. 371-400, Academic Press, New York, 1988.

Creager, K. C., Anisotropy of the inner core from differential travel times of the phases PKP and PKIKP, Nature, 356, 309-314, 1992.

Dahlen, F. A. and J. Tromp, Theoretical Global Seismology, 619-624, Princeton Univ. Press, New Jersey, 1998.

Dziewonski, A. M. and D. L. Anderson, Preliminary reference Earth model, Phys. Earth Planet. Int., 25, 297-356, 1981.

Dziewonski, A. M. and M. Ishii, A three parameter model of inner core anisotropy, IAGA-IASPEI Joint Scientific Assembly, Abstracts, 37, 2001.

Giardini, D., X.-D. Li, and J. H. Woodhouse, Three-dimensional structure of the Earth from splitting in free oscillation spectra, Nature, 325, 405- 
411, 1987.

Gilbert, F. and A. M. Dziewonski, An application of normal mode theory to the retrieval of structural parameters and source mechanisms from seismic spectra, Phil. Trans. Roy. Soc. Lond., Ser. A, 278, 187-269, 1975.

Masters, G. and F. Gilbert, Structure of the inner core inferred from observations of its spheroidal shear modes, Geophys. Res. Lett., 8, 569-571, 1981.

Poupinet, G. R., R. Pillet, and A. Souriau, Possible heterogeneity of the Earth's core deduced from PKIKP travel times, Nature, 305, 204-206, 1983.

Ritzwoller, M., G. Masters, and F. Gilbert, Observations of anomalous splitting and their interpretation in terms of aspherical structure, J. Geophys. Res., 91, 10203-10228, 1986.

Romanowicz, B. A. and L. Breger, Anomalous splitting of core sensitive normal modes: is inner core anisotropy the cause?, EOS Trans. AGU, 80, F17, 1999.

Romanowicz, B. A., L. Breger, and H. Tkalcic, Core sensitive mode and body wave data: possible alternatives to inner core anisotropy, EOS Trans. $A G U, \mathbf{8 1}, \mathrm{S} 314,2000$.

Saito, M., DISPER80: A subroutine package for the calculation of seismic normal mode solutions, in Seismological Algorithms: Computational Methods and Computer Programs, edited by D. J. Doornbos, pp. 293319, Academic Press, New York, 1988.

Sato, Y., Soft core spectrum splitting of the torsional oscillation of an elastic sphere and related problems, Bull. Earthq. Res. Inst., 42, 1-10, 1964.

Takeuchi, H. and M. Saito, Seismic surface waves, in Seismology: Surface Waves and Free Oscillations, Methods in Computational Physics 11, edited by B. A. Bolt, pp. 217-295, Academic Press, New York, 1972.

Tromp, J., Support for anisotropy of the Earth's inner core, Nature, 366, 678-681, 1993.

Tromp, J. and E. Zanzerkia, Toroidal splitting observations from the great 1994 Bolivia and Kuril Islands earthquakes, Geophys. Res. Lett., 22, 2297-2300, 1995.

Widmer, R., G. Masters, and F. Gilbert, Observably split multiplets_-Data analysis and interpretation in terms of large-scale aspherical structure, Geophys. J. Int., 111, 559-576, 1992.

Wiggins, R. A., A fast, new computational algorithm for free oscillations and surface waves, Geophys. J. R. Astron. Soc., 47, 135-150, 1976.

Woodhouse, J. H., The calculation of the eigenfrequencies and eigenfunctions of the free oscillations of the Earth and Sun, in Seismological Algorithms: Computational Methods and Computer Programs, edited by D. J. Doornbos, pp. 321-370, Academic Press, New York, 1988.

Woodhouse, J. H., D. Giardini, and X.-D. Li, Evidence for inner-core anisotropy from splitting in free oscillation data, Geophys. Res. Lett., 13, 1549-1552, 1986.

S. Tsuboi (e-mail: tsuboi@yokohama-cu.ac.jp) and M. Saito 